\title{
Highlights from the observations of the Milky Way with H.E.S.S.
}

\author{
Nukri Komin* \\ School of Physics, University of the Witwatersrand, Johannesburg, South Africa \\ E-mail: nukri.komin@wits.ac.za
}

\section{for the H.E.S.S. Collaboration ${ }^{\dagger}$}

The High Energy Stereoscopic System (H.E.S.S.) has observed the Milky Way at very-high energies (VHE, E > $100 \mathrm{GeV}$ ) since its inauguration in 2004. As sytematic survey of the Milky Way was completed and results were published in 2018. This survey revealed 78 VHE sources. While some of the objects can be identified as pulsar wind nebulae (PWNe), supernova remnants (SNRs) or gamma-ray binaries, the majority of the sources have no firm association with objects known from other wavelengths.

Since then the science focus has shifted towards more detailed studies of the morphology, spectrum and time variability of individual sources. The gamma-ray morphology of resolved sources (namely PWNe and SNRs) can be compared with results from other wavelengths (X-rays, radio) allowing the study of the parent particle distribution and their propagation. An improvement of the reconstruction and analysis pipeline allows even the spatial resolution of the Crab Nebula. The investigation of the high-energy end of the spectra of hard-spectra sources will help to identify PeVatrons, the sources of the Galactic Cosmic Rays with energies up to the knee. Time-resolved measurements investigate the periodic emission from gamma-ray binaries at time-scales of years down to days, study pulsed emission from the Vela pulsar with a period of $89 \mathrm{~ms}$ and searches for transient emission such as flares of binaries or supernova explosions.

In this talk I will present highlights of the results obtained from the observations of the Milky Way with H.E.S.S. in the last two years. I will put these results into a broader context and discuss the implications for future VHE gamma-ray observations.

36th International Cosmic Ray Conference -ICRC2019-

July 24th - August 1st, 2019

Madison, WI, U.S.A.

\footnotetext{
*Speaker.

${ }^{\dagger}$ https://www.mpi-hd.mpg.de/hfm/HESS/pages/collaboration/
} 